\title{
Dental Incremental Lines in Sika Deer (Cervus nippon); Polarized Light and Fluorescence Microscopy of Ground Sections
}

\author{
Yasuko M. IINUMA ${ }^{1}$, Shu TANAKA ${ }^{2}$, Kenzo KAWASAKI $^{2)}$, Toshiaki KUWAJIMA ${ }^{3)}$, Hidehiko NOMURA ${ }^{3)}$, \\ Masatsugu SUZUKI ${ }^{1)}$ and Noriyuki OHTAISHI ${ }^{1)}$ \\ ${ }^{1)}$ Laboratory of Wildlife Biology, Graduate School of Veterinary Medicine, Hokkaido University, Kita-ku, Sapporo 060-0818, \\ ${ }^{2)}$ Department of Anatomy, Tsurumi University School of Dental Medicine, Tsurumi-ku, Yokohama 230-8501 and ${ }^{3)}$ Technical Laboratory \\ Group, Graduate School of Science, Hokkaido University, Kita-ku, Sapporo 060-0810, Japan
}

(Received 8 October 2003/Accepted 19 January 2004)

ABSTRACT. Periodic growth incremental lines are found universally in dental hard tissues. This periodicity theoretically allows for estimation of age, even in days, which would be useful in studies of wild animals. In the present study, enamel and dentin increments of the sika deer (Cervus nippon) were observed in ground sections with a polarized light microscope, and their periodicity was examined by the use of a chronological labeling method with fluorochromes. Enamel increments occurred at a mean interval of 10.6 (SD=1.5) $\mu \mathrm{m}$, and mean spacing of dentin increments was $17.3(\mathrm{SD}=1.8) \mu \mathrm{m}$. Fluorochromic marking revealed that incremental lines form each day in enamel and almost every second day in dentin. The fluorescence-labeled lines suggest that enamel formation of the first $\mathrm{m}$ olar is complete by the age of 5 months. Due to its longer interval of incremental lines and longer term of formation, we conclude that dentin is more suitable than enamel for day-age estimation in sika deer. Experimental confirmation of incremental growth periodicity in various species can improve the reliability of use of tooth increments for age estimation and life history reconstruction. KEY WORDS: age estimation, circadian, incremental line, periodicity, tooth.

J. Vet. Med. Sci. 66(6): 665-669, 2004

Incremental lines that demonstrate regular growth are universal in dental hard tissue. Increments of enamel and dentin reflect systematical metabolic rhythms [11, 21], and many studies have revealed circadian patterns and even subdaily (infradian or ultradian) patterns in various animals [e.g. 3, 7, 17, 18, 22]. This periodicity theoretically allows for estimation of age, even in days; incremental lines have been used to estimate day-age at death in some anthropological studies [1, 4, 5, 9]. The ecological relevance of these increments has also been investigated, and has been used to reconstruct individual life histories $[6,12,13,15]$. Thus, a better understanding of tooth incremental lines may facilitate studies of wild animals where continuous observation is difficult.

In a previous study [10], we showed the daily periodicity of incremental lines in dentin of deciduous teeth from sika deer (Cervus nippon) by the use of decalcified cross sections stained by Bodian's method. However, those findings suggest that permanent teeth are more appropriate than deciduous teeth for age estimation from dentin increments because of differences in dentin deposition rate [10]. In addition, because tooth enamel is eroded by decalcification, enamel increments could not be observed. Most of the day-age estimations and reconstructions of life histories were performed with the Retzius lines, which are long-period lines in enamel. Therefore, in the present study, we prepared longitudinal undecalcified ground sections from first molars (permanent tooth) of sika deer, and examined the incremental lines in enamel and dentin.

\section{MATERIALS AND METHODS}

The animals used were originally described by Iinuma $e t$ al. [10]. Six sika deer fawns were obtained from the captive herd of Ashoro district in eastern Hokkaido. For chronological labeling, each deer was given alternate injections of the vital stains oxytetracycline and calcein, at 13- to19-day intervals between August and October 1998. The detailed injection histories are shown in Fig. 1.

First molars were extracted and fixed in $10 \%$ buffered formalin for 1 week, then air dried and embedded in resin (Epofix: Marumoto Struers K. K., Tokyo, Japan) in a vacuum desiccator, and polymerized at room temperature for 1 day or longer. The polymerized tooth was cut longitudinally with a diamond-edged saw through the mesial and distal cusps in the bucco-lingual plane (Fig. 2). After grinding to remove scratches, the cutting plane was dried and bonded to a glass slide with epoxy glue. The specimen was sectioned at a thickness of 300 to $400 \mu \mathrm{m}$ with a Crystal Cutter NOVA (Maruto Instrument Co., Ltd., Tokyo, Japan), and the sections were ground to a thickness of 60 to $80 \mu \mathrm{m}$ with descending grits of carborundum (600-4000 grit) with paraffin oil. Bonding the specimen to a glass slide made it easier to prepare a uniformly thin section. To avoid expansion of tooth structure from water absorption, oil was used as the grinding medium. After the specimen was rinsed well in paraffin oil, it was coated with nail polish for microscopy.

Sections were examined with a polarized light and fluorescence microscope (BX60: Olympus Co., Ltd., Tokyo, Japan). Using an ocular micrometer, the spacing of increments was measured along the enamel rods or dentinal tubules, because they mark the path of growth. Increments 


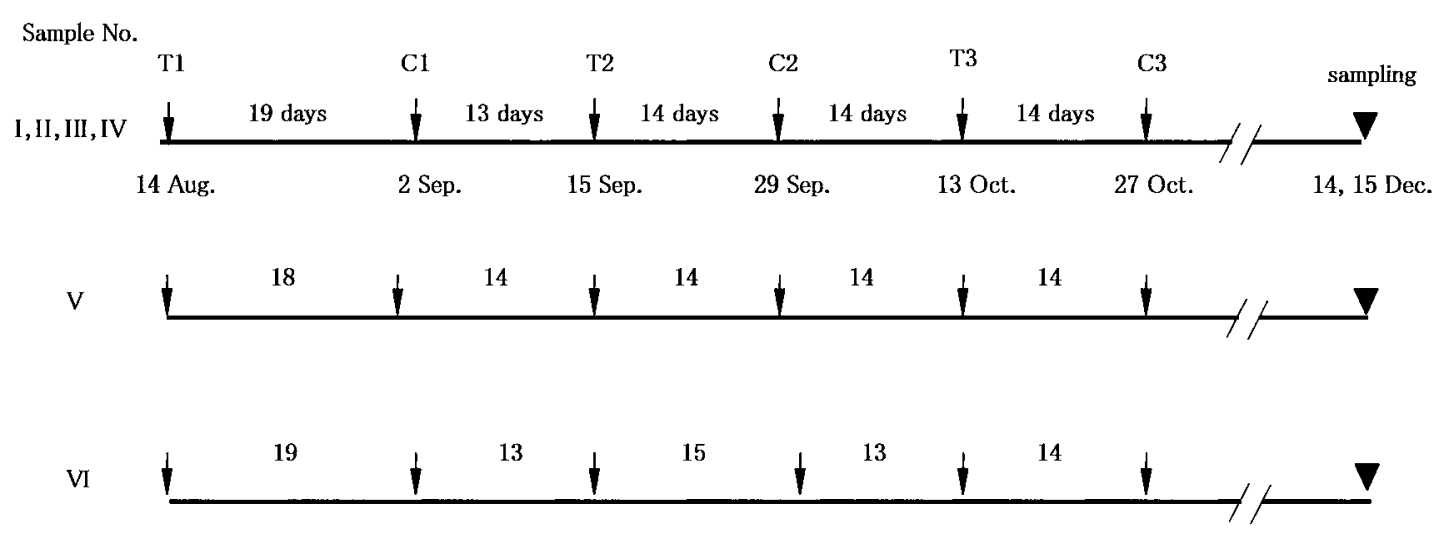

Fig. 1. Injection histories for the chronological labeling method. T1: the first injection of tetracycline, $\mathrm{C} 1$ : the first injection of calcein, T2: the second injection of tetracycline, C2: the second injection of calcein, T3: the third injection of tetracycline, C3: the third injection of calcein.

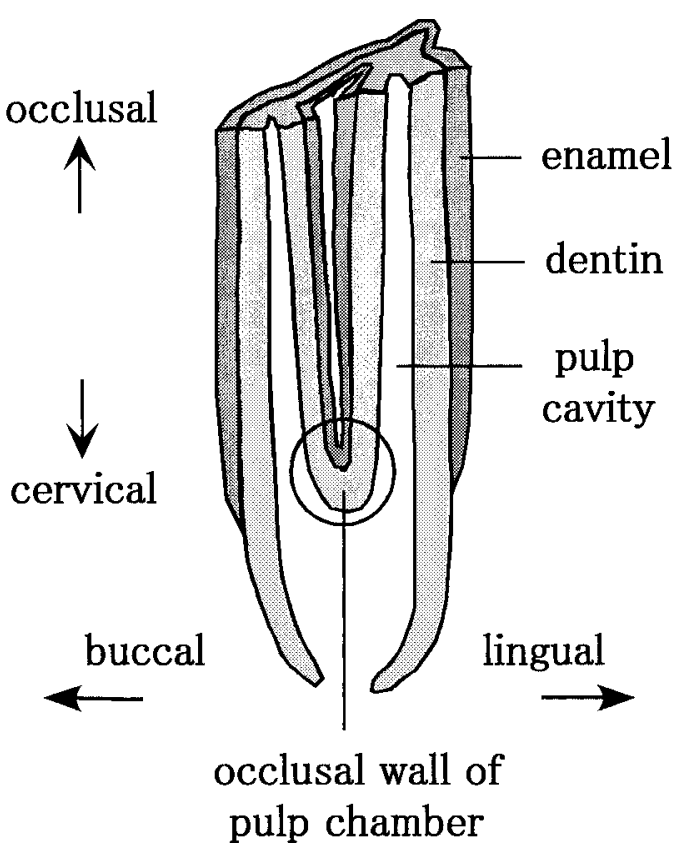

Fig. 2. Diagram of molar (longitudinal plane) showing the term of part of tooth. Both crown and root cementum are omitted.

between labeling lines were counted in superimposed photomicrographs taken with a high-resolution digital camera (HC-2000: Fuji Photo Film Co., Ltd., Tokyo, Japan). First, we photographed the incremental lines in polarized light. Without altering the field of view, the microscope was switched to fluorescent mode (blue-violet light), and oxytetracycline and calcein labeling lines were recorded. Images were transmitted to a computer and composed with image editing software (Adobe Photoshop: Adobe Systems, Inc., 1989-1998). The measurements and the counting were conducted in areas of the tooth where increments were distinctly visible, and repeated at least three times for each slide. To test the accuracy of this superimposition method, both polarized and fluorescent illuminators were used simultaneously with the section showing sufficiently intense fluorescence. It was possible to observe both increments and labeling lines at the same time. The increments between labeling lines on this image corresponded with those in the superimposed image.

\section{RESULTS}

Enamel structures: Minute linear increments ran oblique to the enamel-dentine junction (EDJ). Schreger bands appeared distinctly as light and dark bands running from the EDJ to the enamel surface (Fig. 3b). The increments recurred at an average interval of $10.6 \mu \mathrm{m}$ (minimum, 8.1 $\mu \mathrm{m}$; maximum, $14.7 \mu \mathrm{m}$ ), and the interval tended to increase from EDJ to enamel surface. We observed some calceinlabeled lines in cervical enamel, but did not observe oxytetracycline-labeled lines under fluorescent light. The number of calcein-labeled lines differed among the sites of tooth and among animals. The greatest number of calcein-labeled lines in enamel was observed in the disto-buccal area of the tooth, where two fawns had 1 labeled line, two fawns had 2 labeled lines, and one fawn had 3 labeled lines. When only 1 or 2 calcein-labeled lines were observed, we estimated the injection history of each line based on fluorescence-labeled lines in dentin (Table 1). The number of incremental lines between fluorescence-labeled lines could only be counted in one sample, in which the number of lines was almost equivalent to the number of days between labeling injections (Fig. 3 ). The mean rate of enamel deposition, which was calculated by dividing the distance between calcein-labeled lines by the interval (days) between injections, was 11.2 $(\mathrm{SD}=2.5) \mu \mathrm{m}$ per day. There was very close agreement between spacing of incremental lines and daily deposition rate (Table 1). Other increments (long-period lines) were not distinctly visible.

Dentin structures: In the crown dentin, we observed wider incremental lines, which crossed the dentinal tubules 

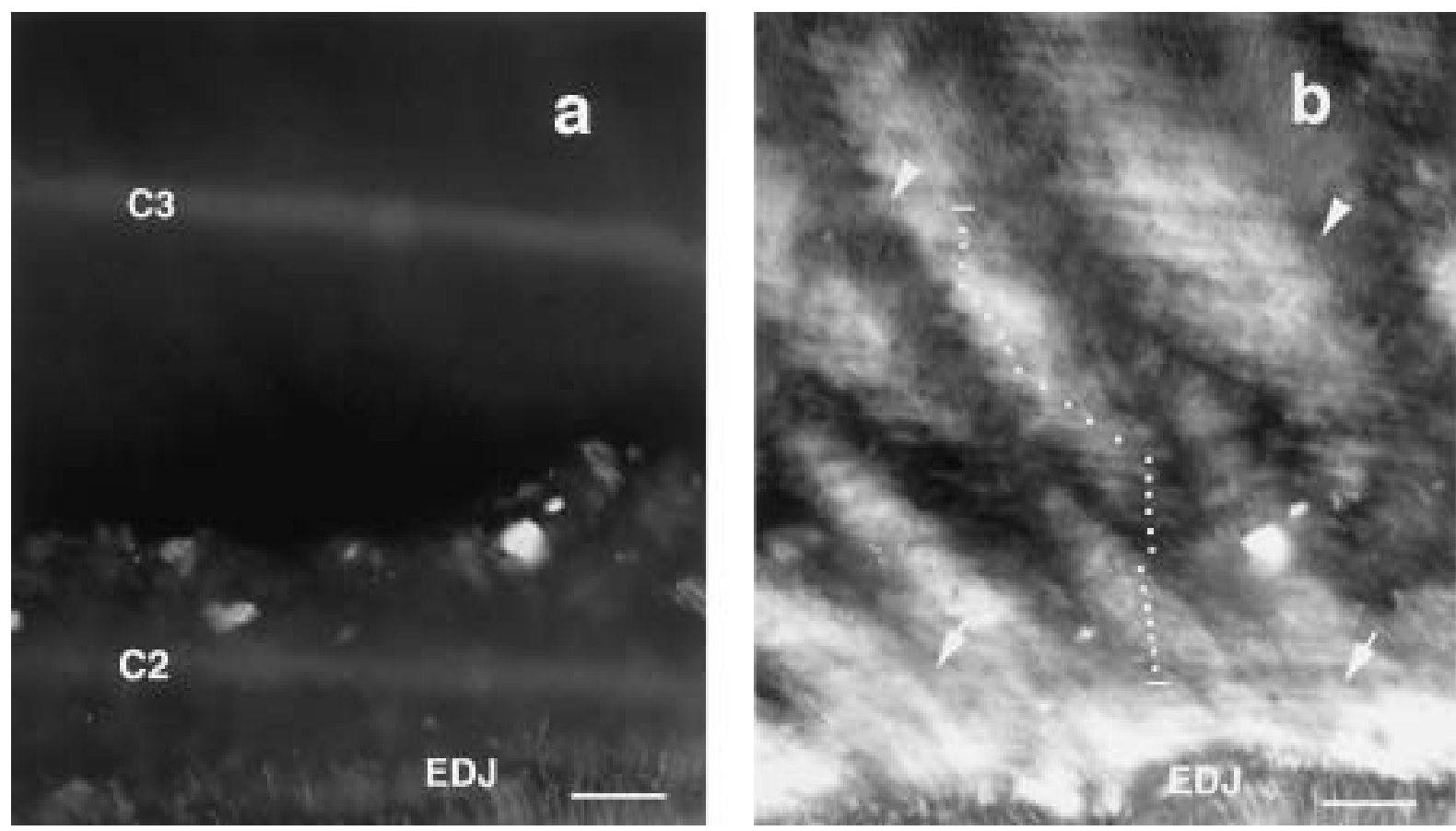

Fig. 3. Comparison of chronological labeling lines and incremental lines in enamel. Fluorescent micrograph (a) and polarized micrograph (b) of the same view observed in sample number VI. The interval day between the second and the third calcein injections was 27 days. a: diffuse fluorescence-labeled lines by calcein are observed, but fluorescence-labeled lines by oxytetracycline are not recognized in enamel. b: Schreger bands appear as light and dark bands running from enamel-dentin Junction (EDJ) to enamel surface ( $t$ op of the figure), and minute linear increments crossing enamel rods obliquely (dots) run left side to right side of the field view . Arrows and arrow heads correspond to $\mathrm{C} 2$ and $\mathrm{C} 3$ in (a) respectively, and 27 increments are counted between calcein-labeled lines. Scalebar = $50 \mu \mathrm{m}$. C2: labeled line by the second calcein injection. C3: labeled line by the third calcein injection. Dot: incremental line. B ar: incremental line labeled by calcein.

Table 1. Values of each individual

\begin{tabular}{|c|c|c|c|c|c|c|c|}
\hline \multirow[b]{2}{*}{ Sample no. } & \multicolumn{4}{|c|}{ Enamel } & \multicolumn{3}{|c|}{ Dentin } \\
\hline & $\begin{array}{c}\text { Spacing of } \\
\text { increments } \\
\text { Mean } \pm \text { SD }(\mu \mathrm{m})\end{array}$ & $\begin{array}{c}\text { Daily } \\
\text { deposition rate } \\
\text { Mean } \pm \text { SD }(\mu \mathrm{m})\end{array}$ & $\begin{array}{l}\text { Periodicity of } \\
\text { increments }{ }^{\text {b) }} \\
\text { (day) }\end{array}$ & $\begin{array}{c}\text { Observed } \\
\left.\text { calcein lines }^{\mathrm{c}}\right)\end{array}$ & $\begin{array}{c}\text { Spacing of } \\
\text { increments } \\
\text { Mean } \pm \text { SD }(\mu \mathrm{m})\end{array}$ & $\begin{array}{c}\text { Daily } \\
\text { deposition rate } \\
\text { Mean } \pm \mathrm{SD}(\mu \mathrm{m})\end{array}$ & $\begin{array}{l}\text { Periodicity of } \\
\text { increments }{ }^{\text {b) }} \\
\text { (day) }\end{array}$ \\
\hline I & $10.4 \pm 1.0$ & $11.5 \pm 2.3$ & - & $\mathrm{C} 1, \mathrm{C} 2$ & $16.7 \pm 1.4$ & $6.4 \pm 0.7$ & 2.2 \\
\hline II & $10.4 \pm 1.2$ & - & - & $\mathrm{C} 1$ & $15.8 \pm 1.1$ & $7.7 \pm 1.6$ & 2.3 \\
\hline III & $10.6 \pm 1.7$ & - & - & $\mathrm{C} 1$ & $20.2 \pm 1.2$ & $8.1 \pm 0.8$ & 2.3 \\
\hline IV & $11.1 \pm 1.9$ & - & - & broken & $16.9 \pm 0.9$ & $7.4 \pm 0.6$ & 2.4 \\
\hline V & - & $8.9 \pm 2.2$ & - & $\mathrm{C} 1, \mathrm{C} 2$ & - & - & 2.3 \\
\hline VI & $11.3 \pm 1.8$ & $12.1 \pm 2.4$ & 1.0 & $\mathrm{C} 1, \mathrm{C} 2, \mathrm{C} 3$ & - & - & 2.2 \\
\hline Average & $10.6 \pm 1.5$ & $11.2 \pm 2.5$ & 1.0 & & $17.3 \pm 1.8$ & $7.4 \pm 1.2$ & 2.3 \\
\hline
\end{tabular}

a) Calculated by dividing the distance between labeling lines by the interval (days) between injection.

b) Calculated by dividing the interval days between labeling injections by the number of increments between labeling lines.

c) C1: labeled line by the first injection, C2: labeled line by the second injection, C3: labeled line by the third injection.

_, No data, because of absence of labeling line or visible increments.

and ran parallel to the outline of the pulp cavity. The most distinctly visible incremental lines were observed in the cervical area and in the occlusal wall of the pulp chamber (Fig. 2). Incremental lines appeared distinctly at the outer (EDJ side) wall in the occlusal dentin, and other increments were visible at the inner (pulp side) wall in the cervical dentin. Spacing of incremental lines varied considerably within a tooth; in the cervical region, the mean value was $17.3 \mu \mathrm{m}$ (minimum, $14.8 \mu \mathrm{m}$; maximum, $21.2 \mu \mathrm{m}$ ). In all animals, the number of fluorescence-labeled lines was in accord with the injection time. From 5 to 8 incremental lines were observed between adjacent labeled lines, and the periodicity of the increments was calculated as 2.3 days (Fig. 4). In the cervical region, the mean rate of dentin deposition was 7.4 $(\mathrm{SD}=1.2) \mu \mathrm{m}$ per day. The spacing of incremental lines was approximately equivalent to twice the value of the daily 

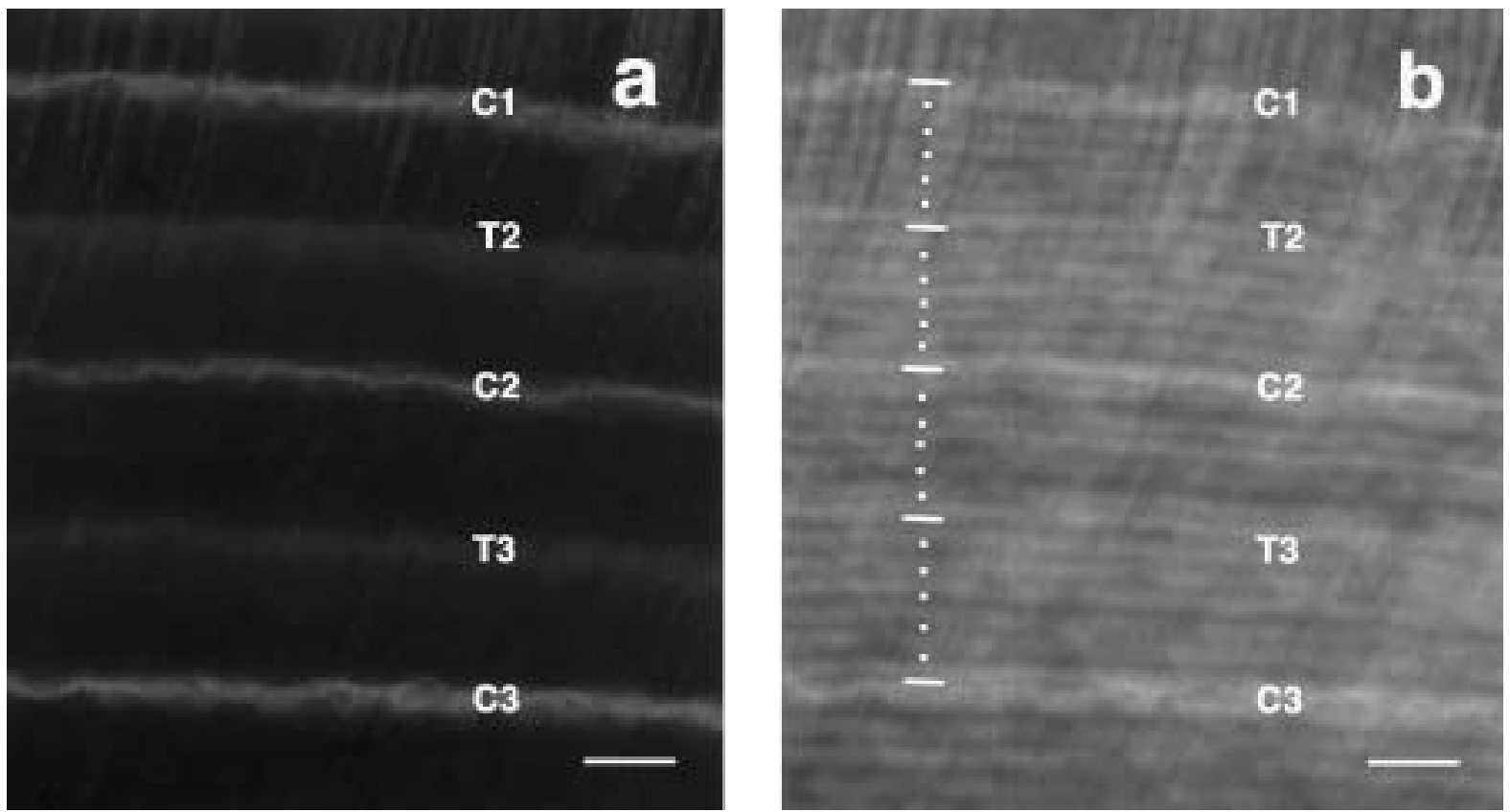

Fig. 4. Comparison of chronological labeling lines and incremental lines in dentin. Fluorescent micrograph (a) and superimposed images of polarized and fluorescent micrographs (b) of the same view observed in sample number I. The injection history of this sample shows 13-14-14-14 day interval. a: the number of fluorescence- labeled lines is in accord with the injection times. Tetrac ycline labels the lines more weakly and more diffusely. b: Incremental lines cross the dentinal tubules. Although tetracycline-labeled lines are not visible distinctly by superimposition, each labeling line is identical with figure (a). Six incremental lines (dots) are counted between each successive labeling line (bars) in this portion. Scale bar $=50 \mu \mathrm{m}$. C1: labeled line by the first calcein injection. T2: labeled line by the second tetracycline injection. C2: labeled line by the second calcein injection. T3: labeled line by the th ird tetracycline injection C3: labeled line by the third calcein injection.

deposition rate (Table 1).

\section{DISCUSSION}

In the present study, the number of the enamel increments between successive labeled lines was consistent with the number of days between injections, suggesting that the incremental lines observed under polarized light reflect the daily periodicity in enamel. The results of comparison between recurrent intervals of increments and daily deposition rates support this suggestion. Similarly, fluorochromic marking revealed that the incremental lines in dentin observed under polarized light are formed at almost every second day.

In the present study, calcein was visible in enamel as diffused fluorescent lines but oxytetracycline was not visible. Unless administered at a very high dose, tetracycline is removed from enamel during maturation $[2,19]$. Therefore, a dose of $40 \mathrm{mg} / \mathrm{kg}$ oxytetracycline should be insufficient for enamel labeling. Although the underlying cause of this calcein staining is unclear, it may have been due to the dose $(6 \mathrm{mg} / \mathrm{kg})$. It is likely that the line labeled by the third calcein injection was not present in enamel in 4 of 5 animals. This suggests that crown formation was completed before the third calcein injection in late October. Because the sika deer is a seasonal breeder with calving time concentrated in
May and June (with some exceptions) [14, 16, 20], the present findings suggest that enamel formation of the first molar is completed at the age of 5 months at the latest. Dentin formation continues for a longer time, until the pulp cavity is filled; thus, dentin incremental lines can be used to determine a wider ranges of ages than enamel incremental lines. Additionally, the long-period lines in enamel were indistinct and dentin increments have a longer period, suggesting that dentin is more suitable for age estimation in sika deer than enamel. As the dentin developed, the area showing countable increments was shifted: in the occlusal area, distinct incremental lines appeared at the EDJ side; in the cervical area, countable increments were visible at the pulp side. To estimate age, all the increments from birth to death must be counted. In on previous study, in which cross sections of deciduous teeth were used, the increments were not distinctly visible near the pulp cavity [10]. Changing the counting site on longitudinal sections of permanent teeth may resolve this problem and allow estimation of age in days.

The incremental growth tracks represent an internal record. Incremental lines have been used in some studies and led to interesting results, but the nature of this periodicity has been the subject of debate [8]. Macho and Williamson calculated crown formation times and the point at which stress lines form, in histological ground sections of molars 
of bovids [15]. However, their calculations were based on the assumption that enamel cross-striation reflects daily periodicity, as in primates, although this has not been confirmed in bovidae. Experimental confirmation of incremental growth periodicity in various species can improve the reliability of using tooth increments for age estimation and life history reconstruction.

ACKNOWLEDGMENTS. We are grateful to Ms. Y. Tanaka, Dr. M. Yokoyama and the members of the town office of Ashoro District for providing samples. We also thank faculty members of Kyushu University Forests in Hokkaido and the many students of Hokkaido University, Obihiro University of Agriculture and Veterinary Medicine for their support in field working.

\section{REFERENCES}

1. Beynon, A. D., Dean, M. C. and Reid, D. J. 1991. Histological study on the chlonology of the developing dentition in gorilla and orangutan. Am. J. Phys. Anthropol. 86: 189-203.

2. Boyde, A. and Reith, E. J. 1982. In vitro histological and tetracycline staining of surface layer rat incisor enamel reflect the cyclical nature of the maturation process. Histochemistry 75 341-351.

3. Bromage, T. G. 1991. Enamel incremental periodicity in the pig-tailed macaque: a polychrome fluorescent labeling study of dental hard tissues. Am. J. Phys. Anthropol. 86: 205-214.

4. Bromage, T. G. and Dean, M. C. 1985. Re-evaluation of the age at death of immature fossil hominids. Nature (Lond.) 317: 525-528.

5. Dirks, W. 1998. Histological reconstruction of dental development and age at death in a juvenile gibbon (Hylobates lar). $J$. Hum. Evol. 35: 411-425.

6. Dirks, W., Reid, D. J., Jolly, C. J., Phillips-Conroy, J. E. and Brett, F. L. 2002. Out of the mouths of baboons: stress, life history, and dental development in the Awash national park hybrid zone, Ethiopia. Am. J. Phys. Anthropol. 118: 239-252.

7. Erickson, G. M. 1996. Daily deposition of the dentine in juvenile alligator and assessment of tooth replacement rates using incremental line counts. J. Morphol. 228: 189-194.

8. FitzGerald, C. M. 1998. Do enamel microstructures have regular time dependency? Conclusions from the literature and a large-scale study. J. Hum. Evol. 35: 371-386.
9. Huda, T. F. J. and Bowman, J. E. 1995. Age determination from dental microstructure in juveniles. Am. J. Phys. Anthropol. 97: 135-150.

10. Iinuma, Y., Suzuki, M., Yokoyama, M., Tanaka, N. Y. and Ohtaishi, N. 2002. Daily incremental lines in sika deer (Cervus nippon) dentine. J. Vet. Med. Sci. 64: 791-795.

11. Klevezal, G. A. 1996. Recording Structures of Mammals. Determination of Age and Reconstruction of Life History. A. A. Balkema. Brookfield.

12. Klevezal, G. A. 2002. Reconstruction of individual life histories of rodents from their teeth and bone. Acta Theriol. 47: 127-138.

13. Klevezal, G. A. and Mina, M. V. 1990. Daily layers and hibernation marks in incisor dentin of Sicista pesudonapaea and some biological remarks. Acta Theriol. 35: 345-356.

14. Koizumi, T. 1992. Reproductive characteristics of female sika deer, in Hyogo Prefecture, Japan. pp 561-563. In: Ongulés/ Unglates 91 (Spitz, F., Janeau, G., Gonzalez, G. and Aulagnier, S. eds.), Société Française pour l'Etude et la Protection des Mammiféres, Toulouse.

15. Macho, G. A. and Williamson, D. K. 2002. The effect of ecology on life history strategies and metabolic disturbances during development: an example from the African bovids. Biol. J. Linnean Soc. 75: 271-279.

16. Miura, S. 1984. Annual cycle of coats changes, antler growth, and reproduction behavior of sika deer in Nara Park, Japan. $J$. Mamm. Soc. Jpn. 10: 1-7.

17. Ohtsuka, M. and Shinoda, H. 1995. Ontogeny of circadian dentinogenesis in the rat incisor. Arch. Oral Biol. 40: 481-485.

18. Rosenberg, G. D. and Simmons, D. J. 1980. Rhythmic detinogenesis in the rabbit incisor: circadian, ultradian, and infradian periods. Calcif. Tissue Int. 32: 29-44.

19. Suga, S. and Murayama, Y. 1965. Microradiographical. ${ }^{45} \mathrm{Ca}$ autoradiographical and tetracycline labeling study on the mineralization of guinea pigs enamel. Odontology 53: 154-162.

20. Suzuki, M., Kaji, K., Yamanaka, M. and Ohtaishi, N. 1996. Gestation age determination, variation of conception date, and external fetal development of sika deer in eastern Hokkaido. $J$. Vet. Med. Sci. 58: 505-509.

21. Ten Cate, A. R. 1998. Oral Histology: Development, Structure and Function. 5th ed., C. V. Mosby, Tront.

22. Yilmaz, S., Newman, H. N. and Poole, D. F. G. 1977. Diurnal periodicity of von Ebner growth lines in pig dentine. Arch. Oral Biol. 22: 511-513. 